\title{
OUTPUT FEEDBACK CONTROL OF LINEAR MULTIPASS PROCESSES
}

\author{
E. Rogers and D.H. Owens
}

Centre for Industrial Modelling and Control and Division of Dynamics and Control, University of Strathclyde, Glasgow G1 1XJ, United Kingdom.

\begin{abstract}
An error actuated output feedback controller for a sub-class of linear multipass processes designated as 'differential unit memory' is defined. Further, the design of this controller for closed-loop stability is considered. In particular, a recently developed computationally feasible stability test is used to present some preliminary work on this problem.
\end{abstract}

\section{Introduction}

Multipass processes are a class of dynamic systems characterised [1] by a repetitive action with interaction between successive passes. Industrial examples include [1] longwall coal cutting and metal rolling. Further, these processes can [1] exhibit undesirable characteristics which require unique control action.

Previous work [2] has developed a rigorous stability theory for multipass processes using an abstract representation which includes the class of so-called [1] differential unit memory linear multipass processes as a special case. The members of this class are described by the state-space model.

$$
\begin{aligned}
& \dot{X}_{k+1}(t)=A X_{k+1}(t)+B U_{k+1}(t)+B_{0} Y_{k}(t), X_{k+1}(0)=0 \\
& Y_{k+1}(t)=C X_{k+1}(t), 0 \leq t \leq \alpha, k \geq 0 \\
& X_{k}(t) \in R^{n}, Y_{k}(t) \in R^{m}, U_{k}(t) \in R^{l}
\end{aligned}
$$

Here $Y_{t}(t)$ is the $k$ th pass profile, $X_{+}(t)$ is the $k$ th pass state vector, $U_{k}(t)$ is the kth pass control input and the pass length $\alpha$ is assumed finite.

The definition of, together with conditions for, stability of (1) can be found in [1]. Further, the testing of these conditions has been considered in [3]. This has resulted in a computationally feasible simulation based test. Note, however, that no work has yet been reported on the design of output feedback based control schemes. Consequently this paper defines a so-called current pass error actuated proportional output feedback controller for (1). Additionally, this recently developed simulation based test is used to present some preliminary work on its design for closed-loop stability.

\section{Stability}

The result of this section is based on the so-called [3] associated conventional linear system of (1) defined as

$$
\begin{aligned}
& \dot{X}(t)=A X(t)+B_{0} Y(t), X(O)=0 \\
& W(t)=C X(t) \\
& \text { or } W=L Y \text { where } \\
& (L Y)(t)=C \int_{0}^{t} e^{A(t-\tau)} B_{0} Y(\tau) d \tau
\end{aligned}
$$

Hence, in effect, (2) has been obtained from (1) by setting $B=0$, dropping the pass subscript and ignoring the pass length. Further, (2) is assumed to be controllable, observable and stable. In addition, it is assumed that its step response matrix $W^{1}(t)=C \int_{0}^{t} e^{M 1} B_{0} d t^{1}$ is available from appropriate simulation studies.

To proceed, let $\mathrm{f}$ be a scalar continuous function defined on any finite interval $[0, t]$. Then $N_{r}(f)$ denotes the norm of $f$ on $[0, t]$, i.e.

$$
N_{1}(f) \triangleq\left|f\left(0^{\dagger}\right)\right|+\sum_{j=1}^{k}\left|f\left(t_{j}\right)-f\left(t_{j-1}\right)\right|+\left|f(t)-f\left(t_{k}\right)\right|
$$

where $0=t_{0}<t_{1}<t_{2}<\ldots-$ are the local minima and maxima of $f$ on $[0,+\infty]$ and $k$ is the largest integer satisfying $4 \leq t$. For $t=+\infty$,

$N_{-}(t)=\sup _{t \geq 0} N_{t}(f)=\operatorname{limit}_{t \rightarrow+\infty} N_{t}(f)$

whenever the limit exists. Note also that the computation of (4) and (5) is a simple exercise [4] from graphical display of f.

Suppose, therefore, that (5) is applied to each element in turn of $W^{1}(t)$ and denote the resulting matrix by $\left\|W^{1} \infty\right\|_{1 p}$. Then the following result, for a proof see [3], constitutes a sufficient condition for stability of (1) where $r($.$) denotes the$ spectral radius.

THEOREM 1: (1) is stable if

$$
r\left(\mid W^{1} \propto d l p\right)<1
$$

The testing of (6) for a given example is straightforward [3], consisting, essentially, of appropriate simulation studies to obtain $W^{1}$ and the subsequent computation of $r\left(\left\|W^{1} \infty\right\|_{p}\right)$. Hence this test is clearly computationally feasible and well suited to inclusion within a computer aided design package.

\section{Ontout Feedback Control}

One approach (for others see [3]) to altering the dynamic characteristics of (1) is to follow standard linear systems theory and employ current pass error actuated output feedback control. Thus a current pass error actuated proportional output feedback controller for (1) takes the form:

$\mathrm{U}_{\mathrm{k}+1}(\mathrm{t})=\mathrm{Ke} \mathrm{e}_{\mathrm{k}+1}(\mathrm{t}), 0 \leq \mathrm{I} \leq \alpha, \mathrm{k} \geq 0$

where $\mathrm{K}$ is an $l \times \mathrm{m}$ real constant matrix, $e_{k+1}(t)=r_{k+1}(t)$ $Y_{k+1}(t)$ is the current pass error vector, and $r_{k+1}(t) \in R^{m}$ represents desired behaviour on pass $k+1$. Suppose also that (7) is applied to (1). Then it follows immediately that the resulting closed-loop system is stable if theorem 1 holds for 
the linear operator defined by substituting A-BKC for A in (3).

Consider now the problem of designing (7) to stabilise (1). Then a fundamental question to be answered is when, and under what conditions, does such a stabilising control law exist. This is termed [3] the existence problem for (7) applied to (1) and its solution in the general case could prove a formidable task. For one special case, however, the following result provides a solution.

THEOREM: Suppose that $\mathrm{m}=l$ and that the matrices $\mathrm{A}, \mathrm{B}$, $B_{0}$ and $C$ are given (after use of a state transformation if appropriate [3]) by $A=-A_{0}{ }^{-1} A_{1}, B=A_{0}{ }^{-1}, B_{0}=L_{\text {and }}$ and $=I_{\text {n }}$ respectively, where $A_{0}$ and $A_{1}$ are real constant matrices. Suppose also that $A_{0}^{-1} A_{1}$ has a diagonal canonical form and set

$K=\rho A_{0}-A_{1}$

where $\rho$ is a positive real scalar. Then $\left\|W_{m}^{1}\right\|_{p}=\frac{1}{\rho} \quad I_{m}$ and hence by theorem 1 the closed-loop system is stable for all choices of $\rho>1$.

Note: Theorem 2 relates to the important practical case when the so-called derived conventional linear system, [3], of (1) has the structure of a multivariable first order lag.

\section{Conclusions}

A current pass error actuated proportional output feedback controller for differential unit memory linear multipass processes has been defined. Further, the design of this controller for closed-loop stability has been considered. In particular, a solution to this problem in one special case has been developed.

\section{References}

[1] Edwards, J.B. and Owens, D.H., 1982, 'Analysis and Control of Linear Multipass Processes' (Wiley Research Press).

[2] Owens, D.H., 1977, 'Stability of Linear Multipass Processes', Proc. I.E.E., 124, (11), pp 1079-1082.

[3] Rogers, E., 1987, 'Feedback and Stability Theory for Linear Multipass Processes', Research Report, The Queen's University of Belfast.

[4] Owens, D.H. and Chotai, A., 1983, 'Robust Controllers for Linear Dynamic Systems Using Approximate Models' Proc. I.E.E., 130 (2), pp45-56. 